$\checkmark$ Research Square

\title{
Analysis of the mental health status and risk factors of the general population in Beijing, China: A cross-sectional study
}

\author{
YUJIN XIE ( 365356971@qq.com) \\ Lei Shi \\ Harbin Medical University \\ Liu Ying \\ Beijing Rehabilitation Hospital of Capital Medical University \\ Di Liu \\ Harbin Medical University \\ Jiao Yang \\ Beijing Rehabilitation Hospital of Capital Medical University \\ Yu Hong \\ Beijing Rehabilitation Hospital of Capital Medical University
}

Beijing Rehabilitation Hospital of Capital Medical University https://orcid.org/0000-0002-4947-0068

Research

Keywords: Mental health, Risk factors, General population in Beijing, Symptom self-rating scale

Posted Date: March 9th, 2020

DOl: https://doi.org/10.21203/rs.3.rs-16353/v1

License: (c) (i) This work is licensed under a Creative Commons Attribution 4.0 International License. Read Full License 


\section{Abstract}

Background: This study sought to understand the mental health status of Beijing's general population, analyze the risk factors that affect it, and provide a scientific basis for promoting the physical and mental health of the general population.

Methods: A cross-sectional design was used. Overall sampling was used to select 6,364 people who had undergone a physical examination from January to December 2018 and volunteered to participate in an assessment of their mental health. We used the SCL-90 to establish the current normal mental health model of the general population in Beijing. After using the PEM System to export and automatically generate Excel files, SPSS 19.00 was utilized for statistical analysis. Descriptive statistical analysis methods were used to analyze the mental health status of the respondents and the mean and standard deviation of each factor. A t-test was used to compare the mental health of the general population in Beijing with the Chinese norm in 1986. Multiple linear regression analysis was used to explore the influencing factors of the mental health of the general population in Beijing.

Results: The average score of each factor of the SCL-90 in Beijing's general population was between 1.28 and 1.75, with no significant peaks. The scores of various factors in the general population in Beijing were relatively close, and their mental health remained stable. The analysis of the demographic variables of gender, education, age, marital status, and monthly income showed that monthly income $(\beta=-0.027, \mathrm{p}<0.05)$ and gender $(\beta=-0.026, \mathrm{p}<0.05)$ had significant effects on the SCL-90 score of the general population.

Conclusion: The mental health level of Beijing's general population was lower than the Chinese norm in 1986. Male mental health problems were prominent and low-income people were prone to psychological problems. It is imperative to strengthen the construction of a mental health service system and standardized management among the Chinese population.

Strengthening the popularization of mental health knowledge, guiding the Chinese population to cultivate a positive attitude, preventing bad attitudes, encouraging the use of scientific methods to deal with psychological behavioral problems, and early targeted measures can reduce the occurrence of serious psychological problems.

\section{Background}

When the World Health Organization (WHO) was founded in 1946, the concept of health was defined in its charter: "Health is a state of physical, psychological and social fulfillment, not just a state free of disease and weakness." In 1990, the WHO defined the concept of "health" as follows: "Health is not only the elimination of debilitating diseases, but also the physical, mental, and social environmental interactions in a normal state" [1]. Having a healthy body is the basis of human survival. Peoples' health not only refers to physical health, but also includes mental health and social adaptability. British psychologist H. B. English [2] pointed out in 1958 that "Mental health is a continuous state of mind. Under this state, the parties can make good adjustments, have vitality, and fully exert their physical and mental potential. This positive and rich state is not only free from mental illness." Thomas Holmes [3], a famous psychologist, proposed in the 1960s that psychosomatic diseases are mainly caused by people's mental health problems. Therefore, it is generally believed that psychology and physiology restrict and influence each other. In 2001, the WHO further defined mental health as a state of health or well-being [4]. Therefore, mental health is an important part of human health [5]. Mental health is the basis of physical and physical health, and it is an indispensable condition for measuring the overall health of an individual. People who have been in a state of fatigue for a long time also have a significantly increased risk of cardiovascular disease, psychological disease, cancer, and other diseases [6].

With the development of the global society and economy and the improvements in medical and health services, the average life expectancy of human beings has continuously increased. Physical health has made considerable progress, but the mental health problems that have followed have not yet been effectively addressed and resolved. At present, global mental health issues have become increasingly prominent. Mental illness seriously damages the quality of life of citizens in both developed and developing countries [7]. The WHO estimates that more than 10 million suicide attempts occur worldwide each year, and 5 of the top 10 diseases that cause the most disability are mental disorders [8]. According to the WHO, by 2020, adolescents with psychological problems worldwide will grow in number by more than $50 \%$. Mental illness will be one of the top five causes of 
adolescent illness, disability, and death [9]. Mental illness is not a separate effect on related patients; it has a wide-ranging impact on the surrounding people and society and even the entire country.

Chinese society is undergoing continuous changes, involving both opportunities and challenges. The living pressure of citizens is gradually increasing, and emotional distress is widespread. According to estimates by the WHO, by 2020, the burden of mental illness in China will rise to a quarter of the total burden of illness [10]. At present, anxiety and depression in Chinese professional groups are quite serious. Depression exists in more than half of the population; $25.60 \%, 23.52 \%$, and $1.58 \%$ had mild, moderate, and severe depression, respectively [11]. In recent years, with the rapid development of China's society and economy, the issue of mental health has received increasing attention, and researchers have begun to pay attention to the current state of the population's mental health. The Baoshan District Center for Disease Control and Prevention in Shanghai conducted a survey of residents' mental health from February to March 2012. It was found that the pressure on residents from social communication, work, and study accounted for $31.4 \%, 29.1 \%$, and $71.8 \%$, respectively, of people's professional psychological service needs [12]. The mental health of the citizens of Chongqing is not good: $81.9 \%$ of them have health problems. Among them, the detection rate of high-knowledge anomaly indicators is more than three times that of ordinary citizens. Psychological tests found that more than $70 \%$ of Chongqing's residents have sub-health mentality, and white-collar workers are the main sub-health group [13]. In 2019, the Institute of Psychology of the Chinese Academy of Sciences released the "Chinese National Mental Health Development Report 2017-2018." Based on a nationwide sample survey, the mental health of Chinese nationals is generally good, but the mental health of the working population deserves attention. The mental health levels of teachers, medical staff, bank employees, and IT industry employees have shown a downward trend year by year, and are lower than the Chinese norm in 1986. High anxiety and stress are common, severe burnout occurs, and there is a huge demand for mental health services [14]. At present, the Chinese use the normal model of the Symptom Checklist 90 (SCL90) scale and compare it with the normal model data of Chinese people in 1986 [15]. However, over the past 30 years, China's economy, culture, living standards and lifestyles, and residents' health have changed dramatically. The mental health of the residents has also changed.

Therefore, in this study, the general population in Beijing was taken as the research object, and the SCL-90 was used to establish the current mental health norms of the general population in Beijing via cluster sampling to understand their mental health. We analyzed the risk factors that affect the mental health of the general population in Beijing and provide a scientific basis for promoting the physical and mental health of the general population.

\section{Methods}

\section{Data collection}

Using the overall sampling method, 6,364 people who had undergone a physical examination at the Beijing Rehabilitation Hospital affiliated with the Capital Medical University from January 2018 to December 2018 and volunteered to participate in the assessment of mental health were selected as the research subjects (enrollment criteria: $18 \leq$ age $\leq 60$ years old; not hearing impaired; volunteered to participate in this study; lived in Beijing for more than 3 years; no missing data). A total of 6,800 questionnaires were distributed and 6,364 were recovered. The effective recovery rate was $93.6 \%$.

\section{Measuring instruments}

The questionnaire survey method required the survey subjects to read the instructions carefully before answering the questions, and the trained medical staff gave on-site, one-on-one guidance to ensure the quality of the survey responses. The questionnaire information included the following: 1) basic personal information on gender, age, marital status, education level, monthly income level, etc. 2) The SCL-90 [16]. The PEM system was used as the platform and the SCL-90 was used as the survey tool. The PEM is the only service system in China that integrates psychological assessment and mental health management. The platform can carry out large-scale mental health assessment of the entire population under the premise of ensuring quality, and it includes the most comprehensive and authoritative psychological assessment scale system based on the SCL-90. Based on a systematic evaluation of the mental health of the general population in Beijing, the platform can also 
perform intelligent expert analysis, multidimensional and multilevel psychological promotion, and psychological intervention. The mental health measurement tool used in the study was the SCL-90, which is a commonly used scale in psychopathology and is widely used in clinical diagnosis. Norms have been established and widely used in different cultures and countries [17]. The scale has a total of 90 items. Its nine subscale dimensions are (1) somatization, (2) obsessive-compulsive, (3) interpersonal-sensitivity, (4) depression, (5) anxiety, (6) hostility, (7) phobic-anxiety, (8) paranoid ideation, and (9) psychoticism [18]. It covers a wide range of psychiatric symptoms, such as sensation, emotion, thinking, consciousness, behavior regarding living habits, interpersonal relationships, eating and sleeping, etc., using a Likert-style five-point scoring method $(0=$ not at all to 4=extremely). It has good reliability and validity [19]. The sum of the 90 individual items can reflect the severity of the disease. Each factor reflects the symptoms of a certain aspect of an individual, and the distribution of symptoms can be understood by the factor scores. The factor score is equal to the total score for each factor divided by the number of items that make up a factor. When an individual's score on a factor is greater than 2 , which is beyond the normal average, the individual is likely to have mental health problems in this area.

\section{Data analysis}

After using the PEM System to export and automatically generate an Excel file, the SPSS 19.00 software was used for statistical analysis of the data. Descriptive statistical analysis methods were used to analyze the mental health status of the respondents and the mean and standard deviation of each factor. A t-test was used to compare the mental health of the general population in Beijing with the Chinese norm in 1986. The multiple linear regression analysis method was used to explore the influencing factors of the mental health of the general population in Beijing. A p-value of $<0.05$ was considered statistically significant.

\section{Results}

\section{Demographics and characteristics}

The average age of the respondents was 39.62 \pm 9.53 , ranging from 19 to 60 years. See Table 1 for basic information such as gender, age group, marital status, education level, and monthly income.

\section{Mental health of the general population in Beijing}

According to the SCL-90 evaluation results, the test population was divided into mental health (all factors averaged $<1.5$ points), mental sub-health (more than 1 factor averaged $\geq 1.5$ points and $<2$ points), mild psychological problems ( 1 or 2 factors were equally divided between $\geq 2$ points and $<3$ points), moderate psychological problems ( 1 or 2 factors were equal $\geq$ 3 points and $<4$ points, or 3 or more factors were equal $\geq 2$ points and $<3$ points), and severe psychological problems (more than 1 factor equaled $\geq 4$ points or more than 3 factors equaled $\geq 3$ points; see Table 2).

\section{SCL-90 scores and comparison of the general population in Beijing}

The average scores of SCL-90 factors in the general population of Beijing ranged from 1.28 to 1.75, and no significant peak was found. The test results were compared with the 1986 norm. Except for the paranoid factors, the average scores of the other factors were all higher than those of the 1986 Chinese norm in the Beijing general population, and the difference was very significant $(p<0.01)$. See Table 3 for details.

\section{Impact of gender on the SCL-90 in Beijing's general population}

A gender comparison of the scores of the general population in Beijing and the 1986 Chinese norm SCL-90 was conducted [15]. The interpersonal sensitivity ( $\mathrm{p} \otimes 0.05$ ), depression ( $\mathrm{x} \otimes 0.05$ ), and paranoid ( $\mathrm{\nabla} \otimes 0.05$ ) factors were statistically significant in male comparisons. The scores of the interpersonal relationship factors were lower than those of the 1986 norm, the scores of the depression factors were higher than those of the 1986 norm, and the scores of the paranoid factors were equal to those of the 1986 Chinese norm. In the comparison of women, it was found that the interpersonal sensitivity ( $p \otimes 0.05$ ) factor was statistically significant, and the score was lower than the Chinese norm in 1986. See Table 4 for details.

Page $4 / 12$ 


\section{SCL-90 scores and comparison of different age groups in Beijing}

The SCL-90 test results of the general population in Beijing at different ages were compared with the 1986 Chinese norm [15]. Except for the 18-29-year-old phobic factor and the 40-49-year-old depression factor, the average scores of the other factors were higher than the 1986 Chinese norm in the general population of Beijing. The difference was very significant (p凶0.01). See Table 5 for details.

\section{Multivariate linear regression analysis of SCL-90 total scores in Beijing's general population}

The SCL-90 total score was used as the dependent variable, and gender, age, marital status, education, and income were used as the independent variables. A multiple linear regression analysis was performed in a stepwise manner. The results showed that the tolerances of the explanatory variables were 0.998 , greater than 0.10 , and the VIFs were 1.002 , less than 2 , suggesting that there was no multicollinearity among the independent variables in the equation. Among them, the regression coefficients of gender and income were negative and less than -0.1, which indicates that the two independent variables had a negative predictive effect on the total score of the SCL-90 $(p<0.05)$. See Table 6 for details.

\section{Discussion}

To illustrate the general population of Beijing's level of mental health, this study compared the SCL-90 scores of the general population in Beijing with the Chinese norm in 1986. The results showed that the overall distribution and severity of the SCL-90 factor scores for the general population in Beijing were basically similar. The average value was between 1.28 and 1.75, and no obvious peaks appeared. Among the top three factors were obsessive-compulsive, interpersonal sensitivity, and hostility. This shows that the scores of the various factors in the general population in Beijing were relatively close, and their mental health has remained stable. Factors such as depression, paranoia, interpersonal sensitivity, and hostility had more discrete scores, but none of them exceed three points, and they have not yet reached medium severity. The general level of mental health in the general population in Beijing is lower than the Chinese norm in 1986. Except for the paranoid factors, the scores of the factors were all higher than the Chinese norm in $1986(t=16.482, p<0.01)$. The forcing factor scored the highest. Its main causes are factors such as fear, a lack of self-confidence, and anxiety caused by huge living stress [20]. Although Beijing has a good working environment and conditions [21], its fast-paced lifestyle and intense social competition pressure have affected the mental health of the general population [22]. The psychological health of the population in developed countries in Europe and the United States is often related to factors such as gender, race, family, economy, and education [23]. However, the characteristics of Chinese politics, culture, and region will determine the particularity of the construction of a mental health service model for the Chinese population.

Compared with the normal model of 1986, it was found that the general population in the 30-39-year-old and 50-60-year-old groups in Beijing scored higher in all factors than the 1986 age group. This shows that with the development of society, the mental health problems in this age group are becoming increasingly serious.

The analysis of the five demographic variables of gender, education, age, marital status, and monthly income in the general population of Beijing showed that monthly income $(\beta=-0.027, p<0.05)$ and gender $(\beta=-0.026, p<0.05)$ had significant effects on the SCL-90 score of the general population.

From the perspective of gender, the general mental health level of the female general population was better than that of the male general population $(\beta=-0.026, p \otimes 0.05)$. The male general population scored higher in interpersonal relationships, hostility, terror, paranoia, and psychotic factors than females ( $\mathrm{p} \llbracket 0.05$ ). This may be due to the fact that men need to assume more family responsibilities and obligations, which results in fewer opportunities for them to communicate with people compared to women. With the development of China's productive forces, the improvement of the social environment, and the improvement of people's basic life factors, the pressure on women's lives in the family has been greatly reduced, and their mental health has been effectively promoted [24]. The female general population had higher scores for the somatization, obsession, depression, and anxiety factors than men $(\mathrm{p}<0.05)$. Due to the influences of women's own physiological factors and traditional cultural thinking, women will face greater and greater employment pressure and unfair competition in the face of fierce social

Page 5/12 
competition. Faced with a social environment with a high elimination rate, women are prone to problems such as placing high demands on themselves, the pursuit of perfect work, inner irritability, decreased self-control, and repeated behaviors [21]. If long-term anxiety and depression cannot be properly channeled in time, they will cause a variety of psychological disorders [25]. The scores of women in factors such as anxiety and psychosis in 2018 were higher than those in 1986, which may be related to the more frequent social activities, the improvement of social status, and the increased social division of labor and social responsibilities in China in recent years [26].

From the perspective of income, the general low-income population was prone to psychological problems $(\beta=-0.027, p \otimes 0.05)$, which is consistent with the results of Zhang's study [27]. The results of public opinion surveys conducted by the Beijing Institute of Social Psychology over the years show that income problems, price problems, medical problems, old-age problems, and housing problems are the main sources of life pressure for Beijing residents. These problems bring a greater sense of stress to most people, especially low-income people, so they are more prone to psychological problems. When income reaches a certain level, the individual's self-regulation space may be greater, and their self-assessed mental health level will become better with the increase in income.

\section{Limitations}

The common Chinese population, as used in this study, is often old, and their mental health is affected by many factors. Social changes and other factors are bound to affect the mental health of this population, so the timeliness of norms is an important issue affecting the accuracy of psychological measurements. In addition to sociodemographic factors, mental health factors are also affected by various things such as the medical security system and the temperament of the Chinese population. However, this study only looked at the main influencing factors from a sociodemographic perspective, which is a limitation.

\section{Conclusions}

The mental health level of Beijing's general population was lower than the Chinese norm in 1986. Male mental health problems were prominent and low-income people were prone to psychological problems. It is imperative that we strengthen the construction of mental health service systems and standardized management among the Chinese population, and boost the scientific popularization of mental health knowledge. Guiding the Chinese population to cultivate a positive mentality, preventing bad attitudes, guiding the entire population to use scientific methods to deal with psychological behavioral problems, and carrying out targeted measures early can reduce the occurrence of serious psychological problems.

\section{Declarations}

\section{Acknowledgements}

The authors wish to thank all the participants who so generously gave their time to be part of this study.

\section{Authors' contributions}

YJX conducted calculations, analyzed results, drafted the manuscript, and contributed equally to this work. LS participated in data processing and revised the paper. YL carried out the epidemiologic investigation of this study. DL assisted with the literature review and data collection. YJ and HY are the corresponding authors of the article; they conceived of the study, participated in its design and coordination. All authors approval of the current version of this manuscript for publications.

\section{Funding}

This research received no specific grant from any funding agency in the public, commercial or not-for-profit sectors.

\section{Availability of data and materials}


The datasets used and/or analysed during the current study are available from the corresponding author on reasonable request.

\section{Ethics approval and consent to participate}

This study was approved by the Ethics Committee of Beijing Rehabilitation Hospital of Capital Medical University. We obtained the consent of each participants involved in the research process.

\section{Consent for publication}

This study has obtained consent to publish from the participants to report individual data.

\section{Competing interests}

The authors declare that they have no competing interests.

\section{Author details}

${ }^{1}$ Beijing Rehabilitation Hospital of Capital Medical University, Beijing 100144, China. ${ }^{2}$ Harbin Medical University, Harbin 150081, China.

\section{Abbreviations}

WHO: World Health Organization; SCL-90: Symptom Checklist 90; PEM: Psychological Elevation Management

\section{References}

1. Queiroz EKRD, Waissmann W. Occupational exposure and effects on the male reproductive system. Cadernos De Saúde Pública. 2006; 22(3):485.

2. English HB, English AC. A Comprehensive Dictionary of Psychological and Psychoanalytical Terms. Science. 2006; 128(10):957.

3. Spann M, Molock SD, Barksdale C, Matlin S, Puri R. Suicide and African American Teenagers: Risk Factors and Coping Mechanisms. Suicide Life-Threat. 2006; 36(5):553-68.

4. Qi SY, Xi YJ, Ma X: A review of Chinese research on mental health. Chin J Health Psychol. 2019; 27(6):947-53.

5. Ahola K, Väänänen A, Koskinen A, Kouvonen A, Shirom A. Burnout as a predictor of all-cause mortality among industrial employees: A 10-year prospective register-linkage study. J Psychosom Res. 2010; 69(1):51-7.

6. Zeng LJ, Wu XC.A Survey of the Research into Job Burnout Abroad. J Shenyang Norm Univ (Social Sci Edition).2003; 27(1):81-4.

7. Shumye S, Belayneh Z, Mengistu N. Health related quality of life and its correlates among people with depression attending outpatient department in Ethiopia: a cross sectional study. Health Qual Life Out. 2019; 17:169.

8. Liu HS. A Study of Boxing Highlighting Against Different Display Backgrounds and at Difficulty Levels. Psychol Sci. 2001; 24(4):480-1.

9. Zimet DM, Jacob T. Influences of Marital Conflict on Child Adjustment: Review of Theory and Research. Clin Child Fam Psychol Rev. 2001; 4(4):319.

10. Organization WH. World health report 2002: reducing risks, promoting healthy life. Educ for Health. 2003; $16(16): 230$.

11. Li JM, Wang W. Meta-analysis on the mental state of occupational people. Occup and Health. 2003; 19(11):10-2.

12. Wang L, Cai ZY, Chen T. Investigation about Residents' Mental Health in Baoshan District of Shanghai. Chin J Social Med. 2013; 30(2):112-4. 
13. Zhang ML. Multicenter study on health status of adult residents in Chongqing in 2016. Third Military Medical University. 2017.

14. Yang S. China's first blue book on mental health officially released. Chin J Disaster Med. 2019; 7(3) :145.

15. Jin H, Wu WY, Zhang MY. Preliminary Analysis of SCL-90 Assessment Results of Chinese Normal People. Chin J Nerv Mental Dis. 1986; 12(5):260-3.

16. VDerogatis L R, Unger R. Symptom Checklist-90-Revised. The Corsini encyclopedia of psychol. 2010:1-2

17. Zhang J, Zhang X. Chinese college students' SCL-90 scores and their relations to the college performance. Asian J Psychiatry. 2013; 6:134-40.

18. Derogatis LR, Cleary PA. Confirmation of the Dimensional Structure of the SCL-90: A Study in Construct Validation. J Clin Psychol. 1977; 33:981-9.

19. Carlozzi NE, Long PJ. Reliability and Validity of the SCL-90-R PTSD Subscale. J Interpers Violence. 2008; 23(9):1162-76.

20. Liu GH. Research on the Obsessive-Compulsive Symptoms of College Students. Chin J Health Psychol. 2017; 25(3):330-3,4

21. Chi XY, Wu YQ, Kang RY. Mental health investigation of community general practitioner in Fengtai District of Beijing City. Chin Med Herald. 2014, 11(33):112-5.

22. Zhao JL, Fu AL, Zhang GH, Zhan Q, Liu JW. Relationship between mental health and job burnout in different ethnic occupational population, Xinjiang. Modern Preventive Med. 2017, 44(15):2717-22.

23. Kirk MD. Investigating relationships between spiritual well-being, stress coping skills, and quality of life among African Americans, Native Americans and Latinos. Dissertations and Theses - Gradworks. 2011, 21:162.

24. Shang L, Li Y, Li J, Fan LB, Gu HK. Gender diffierences in effect of psychosocial work environment on health functioning in Chinese urban occupational population. Chin J Public Health. 2008, 24(12):1519-21.

25. Liang CH. A Probe into the Mental Health of Professional Women. Guangxi Social Sci. 2005(12):183-5.

26. Research Group of Theoretical Research Center of Shanxi Administration Institute, Feng JC, Liu N. The Characteristics and Coping Strategies of Chinese Women's Socioeconomic Status--Based on the Data of Shanxi Province in the Third Chinese Women's Social Status Survey. J Chin Academy Of Governance. 2014;(1):86-90.

27. Zhang ML, Gu XH, Zhu J. Analysis of SCL-90 test results of 809 physical examinees. Chongqing Med. 2017; 46(14):196365.

\section{Tables}

Table 1 Demographic characteristics of the whole sample(n=6364) 


\begin{tabular}{|c|c|c|}
\hline Variables & $\mathrm{n}$ & Percentage(\%) \\
\hline \multicolumn{3}{|l|}{ Gender } \\
\hline Male & 3837 & 60.3 \\
\hline Female & 2527 & 39.7 \\
\hline \multicolumn{3}{|l|}{ Age group(years) } \\
\hline $18-29$ & 1085 & 17.0 \\
\hline $30-39$ & 2255 & 35.4 \\
\hline $40-49$ & 1836 & 28.8 \\
\hline $50-60$ & 1188 & 18.7 \\
\hline \multicolumn{3}{|l|}{ Marital Status } \\
\hline Married & 5585 & 87.8 \\
\hline Single/divorced/widowed & 779 & 12.2 \\
\hline \multicolumn{3}{|l|}{ Education level } \\
\hline Junior college or below & 1898 & 29.8 \\
\hline College & 3650 & 57.4 \\
\hline Master or above & 816 & 12.8 \\
\hline \multicolumn{3}{|l|}{ Monthly income(RMB) } \\
\hline ๑3000 & 420 & 6.6 \\
\hline $3000-5000$ & 1421 & 22.3 \\
\hline$\square 5000$ & 4523 & 71.1 \\
\hline
\end{tabular}

Table 2 Mental health of the general population in Beijing

\begin{tabular}{lcl}
\hline Mental health level & N & Percentage(\%) \\
\hline Mental health & 1427 & 22.4 \\
Mental sub-health & 1931 & 30.3 \\
\hline Mild psychological problems & 1230 & 19.3 \\
\hline Moderate psychological problems & 1489 & 23.4 \\
\hline Severe psychological problems & 287 & 4.5 \\
\hline
\end{tabular}


Table 3 Comparison of results obtained by self-assessment of General population of Beijing and norm data $( \pm s)$

\begin{tabular}{|c|c|c|c|c|}
\hline Item & General populat & $\operatorname{Norm}(n=13$ & & $P$ \\
\hline SCL-90 & $138.23 \pm 44.69$ & $129 \pm 38.76$ & 16.482 & 20.000 \\
\hline Average positive factors & $1.54 \pm 0.50$ & $1.44 \pm 0.43$ & 15.398 & 80.000 \\
\hline Somatization & $1.52 \pm 0.52$ & $1.37 \pm 0.48$ & 23.631 & 10.000 \\
\hline Obsessive-Compulsive & $1.75 \pm 0.61$ & $1.62 \pm 0.58$ & 17.470 & 00.000 \\
\hline Interpersonal Sensitivity & $y 1.63 \pm 0.59$ & $1.65 \pm 0.51$ & -2.917 & 0.004 \\
\hline Depression & $1.53 \pm 0.58$ & $1.50 \pm 0.59$ & 4.189 & 0.000 \\
\hline Anxiety & $1.51 \pm 0.55$ & $1.39 \pm 0.43$ & 16.924 & 40.000 \\
\hline Hostility & $1.57 \pm 0.62$ & $1.48 \pm 0.56$ & 12.011 & 10.000 \\
\hline Phobic & $1.28 \pm 0.44$ & $1.23 \pm 0.41$ & 9.820 & 0.000 \\
\hline Paranoid & $1.43 \pm 0.55$ & $1.43 \pm 0.57$ & 0.000 & 0.748 \\
\hline Psychotic Symptom & $1.43 \pm 0.51$ & $1.29 \pm 0.42$ & 22.179 & 90.000 \\
\hline
\end{tabular}

Table 4 Comparison of SCL-90 factor scores of different sexes in Beijing's general population $( \pm s)$ 


\begin{tabular}{|c|c|c|c|c|}
\hline \multirow[t]{3}{*}{ Item } & \multicolumn{2}{|l|}{ Male } & \multicolumn{2}{|l|}{ Female } \\
\hline & General population & of $\operatorname{Norm}(n=724)$ & General population & of Norm $(n=664)$ \\
\hline & \multicolumn{2}{|l|}{ Beijing $(n=3837)$} & \multicolumn{2}{|l|}{ Beijing $(n=2527)$} \\
\hline Somatization & $1.52 \pm 0.53$ & $1.38 \pm 0.49^{* *}$ & $1.52 \pm 0.50$ & $1.37 \pm 0.47^{* *}$ \\
\hline Obsessive-Compulsive & $1.75 \pm 0.62$ & $1.66 \pm 0.61^{* *}$ & $1.76 \pm 0.59$ & $1.59 \pm 0.54^{* *}$ \\
\hline Interpersonal & $1.65 \pm 0.61$ & $1.66 \pm 0.58$ & $1.60 \pm 0.56$ & $1.61 \pm 0.58$ \\
\hline \multicolumn{5}{|l|}{ Sensitivity } \\
\hline Depression & $1.53 \pm 0.58$ & $1.51 \pm 0.60$ & $1.54 \pm 0.57$ & $1.49 \pm 0.56^{* *}$ \\
\hline Anxiety & $1.51 \pm 0.56$ & $1.41 \pm 0.44^{* *}$ & $1.51 \pm 0.53$ & $1.37 \pm 0.42^{* *}$ \\
\hline Hostility & $1.59 \pm 0.65$ & $1.48 \pm 0.56^{* *}$ & $1.54 \pm 0.57$ & $1.45 \pm 0.52^{* *}$ \\
\hline Phobic & $1.29 \pm 0.47$ & $1.23 \pm 0.37^{* *}$ & $1.27 \pm 0.40$ & $1.30 \pm 0.47^{* *}$ \\
\hline Paranoid & $1.46 \pm 0.58$ & $1.46 \pm 0.59$ & $1.37 \pm 0.49$ & $1.41 \pm 0.54^{* *}$ \\
\hline Psychotic symptom & $1.45 \pm 0.53$ & $1.32 \pm 0.44^{* *}$ & $1.40 \pm 0.47$ & $1.26 \pm 0.39^{* *}$ \\
\hline
\end{tabular}

$\left.{ }^{* *} P\right] 0.01$

Table 5 Comparison of SCL-90 factor scores between Beijing ordinary population at different ages and Chinese norms in $1986( \pm s)$ 


\begin{tabular}{|c|c|c|c|c|c|c|c|c|}
\hline & $18-29$ & & $30-39$ & & $40-49$ & & $50-60$ & \\
\hline & General & $\operatorname{Norm}(\mathrm{n}=781)$ & General & $\operatorname{Norm}(n=332)$ & General & $\operatorname{Norm}(n=185)$ & ) General & $\operatorname{Norm}(n=90)$ \\
\hline & population & & population & $f$ & population & of & population & \\
\hline & Beijing $(n=1085)$ & & Beijing $(n=2255)$ & & Beijing $(n=1836$ & & Beijing $(n=1188)$ & \\
\hline ion & $1.44 \pm 0.47$ & $1.34 \pm 0.45^{* *}$ & $1.51 \pm 0.51$ & $1.37 \pm 0.52^{* *}$ & $1.55 \pm 0.52$ & $1.50 \pm 0.50^{* *}$ & $1.58 \pm 0.56$ & $1.42 \pm 0.52^{* *}$ \\
\hline- & $1.81 \pm 0.60$ & $1.69 \pm 0.61^{* *}$ & $1.78 \pm 0.63$ & $1.50 \pm 0.50^{* *}$ & $1.72 \pm 0.59$ & $1.63 \pm 0.53^{* *}$ & $1.70 \pm 0.61$ & $1.46 \pm 0.45^{* *}$ \\
\hline те & & & & & & & & \\
\hline ona & $11.66 \pm 0.58$ & $1.76 \pm 0.67^{* *}$ & $1.65 \pm 0.62$ & $1.47 \pm 0.51^{* *}$ & $1.60 \pm 0.57$ & $1.53 \pm 0.53^{* *}$ & $1.59 \pm 0.58$ & $1.39 \pm 0.37^{* *}$ \\
\hline l & & & & & & & & \\
\hline $\mathrm{n}$ & $1.52 \pm 0.57$ & $1.57 \pm 0.61^{* *}$ & $1.55 \pm 0.62$ & $1.39 \pm 0.52^{* *}$ & $1.52 \pm 0.56$ & $1.51 \pm 0.58$ & $1.51 \pm 0.54$ & $1.36 \pm 0.40^{* *}$ \\
\hline & $1.54 \pm 0.53$ & $1.42 \pm 0.43^{* *}$ & $1.53 \pm 0.57$ & $1.33 \pm 0.42^{* *}$ & $1.49 \pm 0.53$ & $1.41 \pm 0.44^{* *}$ & $1.47 \pm 0.54$ & $1.30 \pm 0.41^{* *}$ \\
\hline & $1.55 \pm 0.60$ & $1.50 \pm 0.57^{*}$ & $1.63 \pm 0.66$ & $1.41 \pm 0.50^{* *}$ & $1.55 \pm 0.59$ & $1.44 \pm 0.53^{* *}$ & $1.53 \pm 0.59$ & $1.34 \pm 0.39^{* *}$ \\
\hline & $1.32 \pm 0.43$ & $1.33 \pm 0.47$ & $1.28 \pm 0.44$ & $1.20 \pm 0.36^{* *}$ & $1.26 \pm 0.42$ & $1.18 \pm 0.34^{* *}$ & $1.30 \pm 0.50$ & $1.12 \pm 0.31^{* *}$ \\
\hline & $1.43 \pm 0.53$ & $1.52 \pm 0.60^{* *}$ & $1.46 \pm 0.58$ & $1.35 \pm 0.53^{* *}$ & $1.40 \pm 0.53$ & $1.84 \pm 0.51^{* *}$ & $1.41 \pm 0.54$ & $1.20 \pm 0.30^{* *}$ \\
\hline & $1.45 \pm 0.49$ & $1.36 \pm 0.47^{* *}$ & $1.44 \pm 0.52$ & $1.20 \pm 0.31^{* *}$ & $1.41 \pm 0.49$ & $1.21 \pm 0.35^{* *}$ & $1.42 \pm 0.51$ & $1.16 \pm 0.32^{* *}$ \\
\hline
\end{tabular}

${ }^{*} P \llbracket 0.05{ }^{* *} P \unlhd 0.01$

Table 6 Multiple linear stepwise regression analysis of factors influencing the SCL-90 score of the general population in Beijing

\begin{tabular}{lllllll}
\hline Ident variable & Assignment & $B$ & $S E$ & $\beta$ & $t$ & $P$ \\
\hline \multirow{r}{*}{ income (RMB) } & $1=\square 3000,2=3000-5000,3=\square 5000$ & -1.974 & 0.933 & -0.027 & -2.117 & 0.034 \\
& $1=$ Male, 2=Female & -2.368 & 1.145 & -0.026 & -2.068 & 0.039 \\
\hline
\end{tabular}

